\title{
Support vector machine for predicting the Indonesia Stock Exchange Composite Index (IDX Composite) using domestic and international economic factors
}

\author{
M.D. Wahono \& Z.A. Husodo \\ Master of Management, Universitas Indonesia, Jakarta, Indonesia
}

\begin{abstract}
Economic crises in the past have raised questions about the validity of the efficient market hypothesis and led to the development of models that can predict the stock price. The efficiency of the stock markets is known to decline, which means that the stock market's performance is unpredictable. One of the developing models is a prediction based on economic components known to affect the IDX composite index and processing it by machine learning techniques. Support vector machines are known to have the ability to handle high-dimensional data and have advantages over other algorithms. To determine what economic components are used, this study begins with identifying the influence of domestic and international economic components on the future IDX composite index using Pearson's correlation coefficient. The identification result explains that eight domestic and six international components have influences on the IDX composite index. Using these components as IDX composite index-influencing factors, this study builds and evaluates a machine learning-based stock index prediction model. This research used two error parameters: mean absolute error (MAE) and root mean square error (RMSE). The SVM model's performance was compared to the most used machine learning algorithm: artificial neural networks (ANN) and the classic algorithm, multiple linear regression (MLR). The prediction results showed that SVM showed the best performance in predicting next day's stock index prices $(t+1)$.
\end{abstract}

\section{INTRODUCTION}

After the global economic crisis, the efficient market hypothesis (EMH) theory began to be questioned. This phenomenon is supported by findings, which state that the efficiency of the American stock market from 1929-2002 varied over time due to events affecting the financial, economic, and social systems, and it was also suggested that in the last ten years, market efficiency tended to decline (Alvarez-Ramirez et al. 2012). This question led to the emergence of various empirical studies relating to predicting stock price movements using a non-classical approach.

Many financial and economic theories have been developed and research has been conducted, which argued that the stock market is influenced by economic factors, including domestic components represented by macroeconomics and international components (Al-Majali and Al-Assaf 2014). The global economic crisis showed that both domestic and international economic conditions affect the stock market (Adesanmi and Jatmiko 2017). Macroeconomics is a component of the economy that affects the stock market in various ways. These things are shown in the conclusions of several studies on the influence of macroeconomic components on the composite stock index conducted in the past (Celebi and Hönig 2019, Demir 2019, Alshammari et al. 2020, Beh and Yew 2020). The use of daily data for the prediction can increase the prediction model's performance because the amount of available data is large, and this large amount of data can be used to maximize the machine learning process.

The machine learning method is considered to have potential in predicting stock prices and can overcome some of the shortcomings of other methods. The machine learning algorithms widely 
used today include artificial neural networks and the classical method, multiple linear regression (Henrique et al. 2019). The support vector machine is another machine learning algorithm that has a great potential in predicting stock prices. SVM has the advantage of the ability to process high-dimensional data. SVM has advantages over ANN, unlike the ANN training process, which requires nonlinear optimization with the potential danger of being stuck at the local minima. Several studies in economics conducted in the past that utilized SVM support this opinion and report ANN and SVM's success in predicting the stock prices (Weng et al. 2017, Zhong and Enke 2017).

This study aims to build a prediction model involving the supporting components based on the potential of machine learning, especially SVM, in predicting and the strong influence of economic components on stock prices. A machine learning method with the SVM algorithm was used, whose performance was compared with those of ANN and MLR. It showed the accuracy of the model assessed in terms of error. SVM predicted the IDX Composite by using historical economic data as the input.

\section{LITERATURE REVIEW}

\subsection{Economic component}

In analyzing the capital market, this study was started by analyzing the economic environment in which the capital market is located, for example, the domestic and international economic environment. The economy of a country depends on the daily business activities carried out by various companies (Alamsyah and Zahir 2018). Changes in economic components tend to influence the condition of the capital market. So investors, in general, will consider and predict future economic conditions before making investment decisions. This consideration is taken because economic growth is primarily determined by macroeconomic components' volatility (Wahyudi et al. 2017). Also, there is a relationship between the value of share prices and India's economic component (Ahmed 2008). This result explains that there is an interplay between stock prices and macroeconomic components. Thus, macroeconomics has a significant role in determining the future of the capital market.

There are various types of macroeconomic data: domestic and international; annual, monthly, daily, and hourly. The majority of the data used are monthly data, such as money supply, inflation rate, interest rates, and others from previous studies. In Indonesia, a study was conducted using an artificial neural network to predict the IDX Composite using macroeconomic variables (Alamsyah and Zahir 2018). In addition to the domestic economic component represented by macroeconomics, this study uses several international components. Four types of economic components are used as an input in this study: exchange rate, government bond, commodity, and foreign stock price index. The exchange rate and government bond are categorized as domestic economic components because they directly impact the economy and reflect economic conditions. Commodity and foreign stock prices are categorized as an international economic component.

\section{METHOD}

\subsection{Data collection}

For this research, the historical data were obtained from online datastreams: Refinitiv Eikon, AsianBondsOnline, and Investing.com. The data obtained are daily data, the period of which is from 2006 to 2019. It is hoped that large amounts of data will increase the number of training sets, which are nearly related to increasing the model's accuracy. The data obtained were still stored separately and contained outliers. The data collected consist of 26 economic components that were filtered. These variables were categorized into four groups: exchange rates, government bonds, commodities, and the foreign stock composite index. 


\subsection{Preprocessing}

The data collected were compiled into one CSV file. At this stage, the data were conditioned or prepared to proceed to the modeling stage. The preparation included winsorizing, which eliminated the negative effect of outlier and feature selection that helped reduce the computational cost of modeling and improved the model's performance. Winsorizing was done by detecting an outlier and replacing the outlier value with another value that had been determined. For feature selection, Pearson's correlation coefficient was used to calculate the correlation between each economic component and IDX composite and to determine which economic components could be predictor variables. Only the economic components that had a strong correlation with the IDX Composite passed the feature selection.

\subsection{Modeling}

The data that passed the preprocessing stage were ready to be processed. Weka is an open-source machine learning software. This software helped in building models for this research. Three models were built, including three algorithms: SVM, ANN, and MLR. Some parameter settings were judgmental, and other parameters were set based on recommendations from references.

\subsection{Evaluation}

An evaluation stage was needed to understand how well the model performs. For this research evaluation, there are two essential components, namely, 10-fold cross-validation and error metric. 10 -fold cross-validation divided the data into two parts, training dataset and testing dataset, and evaluated it. To assess the performance of a predictive model, the metric was needed. The error matrix is considered most appropriate in assessing the regression model's performance, especially for this study, particularly in terms of mean absolute error and root mean square error.

\section{ANALYSIS}

As predictor variables, 15 variables passed the feature selection, including the economic component and the IDX composite. Machine learning models for three algorithms were built using these 15 variables. Table 1 shows the result of the prediction process by three models formed by three different algorithms.

Table 1. Prediction one day ahead result $(t+1)$.

\begin{tabular}{lll}
\hline Parameter & & Domestic + International \\
\hline ANN & MAE & 36.3662 \\
& RMSE & 49.0389 \\
SVM & MAE & 30.8277 \\
& RMSE & 43.5466 \\
MLR & MAE & 30.9643 \\
& RMSE & 43.5186 \\
\hline
\end{tabular}

From Table 1, we can see that ANN's performance is the worst among the three models. This model has the largest error compared to the other models, namely, MAE of 36.3662 and RMSE of 49.0389. For error reading, the MAE error illustrates that the model can predict the IDX Composite with a sizeable average deviation of 36.3662 either negatively or positively. Meanwhile, based on RMSE, the ANN model can predict with the residual magnitude of 49.0389. SVM's performance is better than ANN and almost the same as MLR, with the MAE value of 30.8277, which is 0.1366 higher, and RMSE of 43.5186, which is 0.0280 lower than those of MLR. This test's results provide an initial overview of each model's performance with the tentative conclusion that SVM and MLR 
show a better performance than ANN in predicting the next day IDX Composite Index. The input or predictor variables consist of 14 economic components and 14-year historical IDX Composite Index data. These results also tell us that the combination of the predictor variable and the target variable gives good results. The best model (SVM) predicts with error or deviation of 30.8277 for MAE and 43.5466 for RMSE from the average IDX Composite: 4011.28 (for the 14-year period) of $0.75 \%$ for MAE and of $1.09 \%$ for RMSE.

\section{CONCLUSIONS}

Prediction of the composite stock price index is essential for various economic agents in carrying out their roles. Successful prediction helps the investors build an optimal portfolio and help the government make appropriate policies. This study aims to build an optimal model to predict the IDX composite index using a potential machine learning algorithm, namely, SVM.

In the process, this study attempted to find the best predictor components and focused on the economy's domestic and international components. Based on their correlation with the future IDX composite index, 15 components (economic components and historical IDX composite index) were selected as predictor variables for prediction. From this research, for $t+1$ prediction, we found that SVM and MLR show quite the same performance and are better than ANN. The error magnitude shows that the models can predict the next day IDX Composite well, and SVM is the best model.

\section{REFERENCES}

Adesanmi, A.A. and Jatmiko, D.P., 2017. The impact of macroeconomic variables on an emerging economy stock market: Evidence from Jakarta composite index, Indonesia. International Journal of Economic Perspectives, 11 (2), 665-684.

Ahmed, S., 2008. Aggregate Economic Variables and Stock Markets in India. International Research Journal of Finance and Economics, 14 (14), 1450-2887.

Al-Majali, A.A. and Al-Assaf, G.I., 2014. Long-Run and Short-Run Relationship Between Stock Market Index and Main Macroeconomic Variables Performance in Jordan. European Scientific Journal, 1010 (1010), 1857-7881.

Alamsyah, A. and Zahir, A.N., 2018. Artificial Neural Network for predicting Indonesia stock exchange composite using macroeconomic variables. 2018 6th International Conference on Information and Communication Technology, ICoICT 2018, 0 (c), 44-48.

Alshammari, A.A., Altarturi, B., Saiti, B., and Munassar, L., 2020. The impact of exchange rate, oil price and gold price on the Kuwaiti stock market: A wavelet analysis. European Journal of Comparative Economics, 17 (1), 31-54.

Alvarez-Ramirez, J., Rodriguez, E., and Espinosa-Paredes, G., 2012. Is the US stock market becoming weakly efficient over time? Evidence from 80-year-long data. Physica A: Statistical Mechanics and its Applications, $391(22), 5643-5647$.

Beh, W.L. and Yew, W.K., 2020. Macroeconomic factors and stock markets interdependencies: Evidence from United States and China. Journal of Critical Reviews, 7 (5), 68-74.

Celebi, K. and Hönig, M., 2019. The impact of macroeconomic factors on the german stock market: Evidence for the crisis, pre-and post-crisis periods. International Journal of Financial Studies, 7 (2).

Demir, C., 2019. Macroeconomic determinants of stock market fluctuations: The case of BIST-100. Economies, 7 (1).

Henrique, B.M., Sobreiro, V.A., and Kimura, H., 2019. Literature review: Machine learning techniques applied to financial market prediction. Expert Systems with Applications, 124, 226-251.

Wahyudi, S., Hersugondo, H., Laksana, R.D., and Rudy, R., 2017. Macroeconomic Fundamental and Stock Price Index in Southeast Asia Countries: A Comparative Study. International Journal of Economics and Financial Issues, 7 (2), 182-187.

Weng, B., Ahmed, M.A., and Megahed, F.M., 2017. Stock market one-day ahead movement prediction using disparate data sources. Expert Systems with Applications, 79, 153-163.

Zhong, X. and Enke, D., 2017. Forecasting daily stock market return using dimensionality reduction. Expert Systems with Applications, 67, 126-139. 